The Astrophysical Journal, 341:208-219, 1989 June 1

(C) 1989. The American Astronomical Society. All rights reserved. Printed in U.S.A.

\title{
AMMONIA OBSERVATIONS OF OUTFLOW REGIONS
}

Guillem Anglada, ${ }^{1,2}$ Luis F. Rodríguez, ${ }^{3,4}$ José M. Torrelles, $^{5}$ Robert Estalella, ${ }^{1,2}$ Paul T. P. Ho, ${ }^{3}$ Jorge Cantó, ${ }^{6}$ Rosario López, ${ }^{1,2}$ AND LOURDES Verdes-MONTENEGRO ${ }^{5}$

Received 1988 September 2; accepted 1988 November 10

\begin{abstract}
Using the $37 \mathrm{~m}$ telescope of the Haystack Observatory, we observed the $(J, K)=(1,1)$ ammonia transition toward the suspected exciting sources of 12 regions with molecular or optical outflows: L1448, L1455, L1524 (Haro 6-10), RNO 43, HH 34, HH 38, 43, Haro 4-255 FIR, NGC 2264 (HH 14-4, 6), L43, R CrA, HH 32a (AS 353A), and V1331 Cyg. We detected and mapped ammonia emission in nine of these regions.

In five of the mapped regions, the spatial coincidence of the high-density gas with the objects previously proposed as exciting sources gives support to those identifications. For L1524 (Haro 6-10) and HH 38, 43, we propose a new location for their outflow exciting sources. We detected a radio continuum source and an unusually strong $\mathrm{H}_{2} \mathrm{O}$ maser coinciding with the maximum of the ammonia emission in L1448. This region appears to be the site of very recent star formation.
\end{abstract}

Subject headings: interstellar: molecules - stars: pre-main-sequence

\section{INTRODUCTION}

Energetic mass outflow is a common phenomenon in starforming regions. Observational evidence of this phenomenon comes mainly from the presence of very broad wings in molecular lines (usually CO lines). A high fraction of these outflows is found to have a bipolar morphology. Rodríguez et al. (1982) and Lada (1985) discussed the main characteristics of these molecular outflows. Other important manifestations of the same phenomenon are the Herbig-Haro objects (Schwartz 1983; Cantó 1985) and the presence of optical jets (Mundt 1986).

One of the most difficult aspects of the outflow phenomenon is the search and identification of its power source (usually manifesting itself as an IR or a radio continuum source). Several criteria have been used to locate these exciting sources, e.g., proximity to the center of symmetry, alignments and proper motions of $\mathrm{HH}$ objects, and visible morphological connections (Herbig and Jones 1981; Cohen, Bieging, and Schwartz 1982; Cohen and Schwartz 1983; Pravdo et al. 1985).

Several studies have shown that the exciting sources of the molecular or optical outflows are frequently embedded in highdensity gas, as traced by the ammonia emission, and are located near the position of the peak (Torrelles et al. 1983, 1985b, 1986; Schwartz, Waak, and Smith 1983; Little et al. 1985; Takano et al. 1987; Myers et al. 1988). In this sense, ammonia observations can be an important tool for discriminating between several objects when different criteria point toward different exciting source candidates, or for narrowing down the region where the exciting source could be searched for.

Based upon these arguments, we have selected 12 regions with signs of molecular or optical outflow to be mapped in ammonia around the position of the suspected exciting source.

\footnotetext{
${ }^{1}$ Departament de Física de l'Atmosfera, Astronomia i Astrofísica, Universitat de Barcelona.

${ }^{2}$ Grup d'Astrofisica, Societat Catalana de Física, IEC.

${ }^{3}$ Harvard-Smithsonian Center for Astrophysics.

4 On sabbatical leave from Instituto de Astronomía, UNAM.

5 Instituto de Astrofísica de Andalucía, CSIC.

${ }^{6}$ Instituto de Astronomía, UNAM.
}

In $\S$ II we describe the observations, in $\S$ III we discuss the individual sources, and in $\S$ IV we give our conclusions.

\section{OBSERVATIONS}

The ammonia observations were made during 1985 May and 1986 March-April with the $37 \mathrm{~m}$ radio telescope at Haystack Observatory. ${ }^{7}$ At the frequency of the $(J, K)=(1,1)$ inversion transition of ammonia $(23.7 \mathrm{GHz})$, the beam size is 1.4 , and the beam efficiency at an elevation of $45^{\circ}$ is $\sim 0.32$. We used a cooled $K$ band maser receiver and the 1024 channel digital autocorrelation spectrometer with an effective bandwidth of $6.67 \mathrm{MHz}$. After Hanning-weighting, the spectral resolution was $\sim 0.2 \mathrm{~km} \mathrm{~s}^{-1}$. All of the spectra were corrected for elevation-dependent gain variations and for atmospheric attenuation. The rms pointing error of the telescope was $\sim 15^{\prime \prime}$. The calibrations were made with the standard noisetube method. System temperatures in the range $70-140 \mathrm{~K}$ were obtained.

We observed 12 sources. In all cases, we first made measurements at the positions of a nine-point grid, with full beam separation between points. These grids were centered at the positions given in Table 1. Ammonia was detected and mapped in nine of the sources. Maps of these sources are given in Figures 1 and 4 to 13. Superposed on these figures we also show the position of several indicators of star formation reported in the literature.

In Table 2, we give the spectral line parameters of the ammonia emission at selected positions of the mapped sources. In Table 3, we list the parameters of the ammonia condensations as derived following the procedures explained in the footnotes of the table. The range of these parameters is similar to those found in previous studies of ammonia condensations associated with outflows (Torrelles et al. 1983, 1986).

III. DISCUSSION OF INDIVIDUAL SOURCES

$$
\text { a) } L 1448
$$

Our ammonia map (Fig. 1) traces the core of this cloud located in Perseus. There is good agreement between the elon-

\footnotetext{
${ }^{7}$ Radio astronomy at Haystack Observatory of the Northeast Radio Observatory Corporation is supported by the National Science Foundation.
} 
TABLE 1

SOURCES OBSERVED IN $\mathrm{NH}_{3}(1,1)$

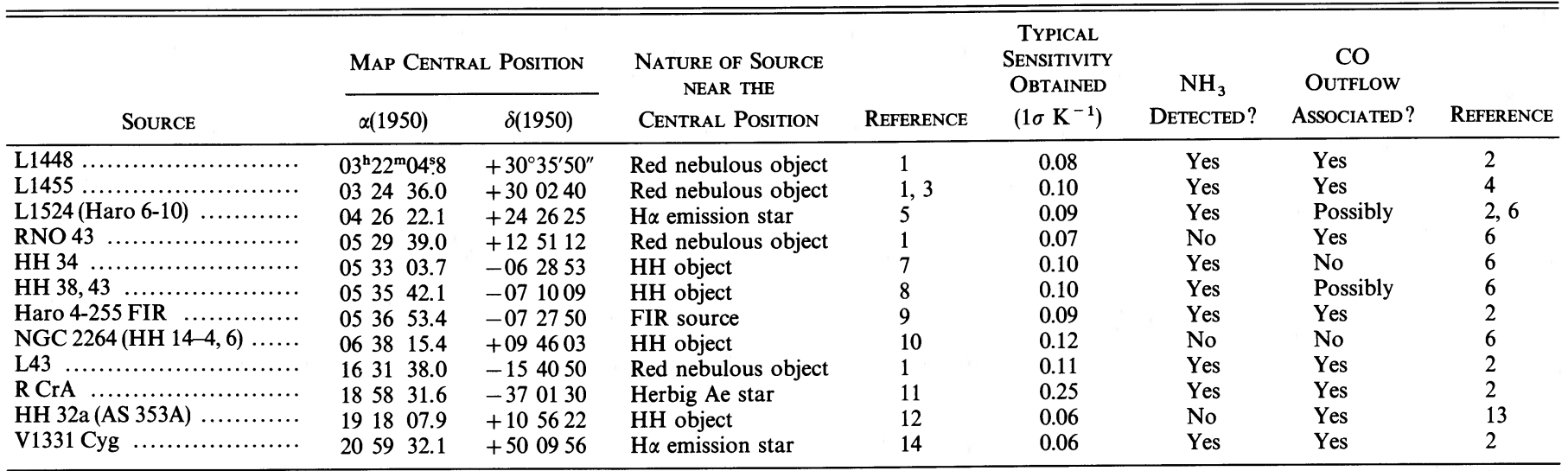

REFERENCES.-11) Cohen 1980; (2) Levreault 1985; (3) Davidson and Jaffee 1984; (4) Goldsmith et al. 1984; (5) Haro, Iriarte, and Chavira 1953; (6) Edwards and Snell 1984; (7) Haro 1959; (8) Haro 1953; (9) Evans, Levreault, and Harvey 1986; (10) Adams, Strom, and Strom 1979; (11) Herbig 1960; (12) Herbig and Kuhi 1963; (13) Edwards and Snell 1982; (14) González and González 1956.

gated morphology of our ammonia map and that of Bachiller and Cernicharo (1986). There are three IRAS sources closely associated with L1448. From west to east, we found IRS 1, coinciding with RNO 13 (Levreault 1985) and located at the edge of the ammonia condensation. The second, IRS 2 (coinciding with RNO 14), seems spatially associated with a relatively low velocity $C O$ outflow $\left(\Delta V=12 \mathrm{~km} \mathrm{~s}^{-1}\right.$ at $0.2 \mathrm{~K}$ level) detected by Levreault $(1985,1988)$. The morphology of this outflow is not well established. Finally, IRS 3 is the brightest of the three sources in the far-IR. It is very close to the peak of the ammonia condensation.

To obtain additional information on the core of this source, we carried out $6 \mathrm{~cm}$ Very Large Array $\left(\mathrm{NRAO}^{8}\right)$ observations of the region during 1987 June 4. At that epoch, the VLA was in the $\mathrm{D}$ configuration, providing an angular resolution of about $10^{\prime \prime}$ at $6 \mathrm{~cm}$. We detected a source at $\alpha(1950)=$ $03^{\mathrm{h}} 22^{\mathrm{m}} 31^{\mathrm{s}} 9, \delta(1950)=30^{\circ} 34^{\prime} 50^{\prime \prime}$, a position that falls inside the error ellipsoid for the source IRAS $03225+3034$ (IRS 3). The source has an integrated $6 \mathrm{~cm}$ flux of $1.3 \mathrm{mJy}$ and appears unresolved for our angular resolution. Assuming it is an optically thin $\mathrm{H}$ II region with an electron temperature of $10^{4} \mathrm{~K}$, we find it requires an ionizing flux of $1.4 \times 10^{43} \mathrm{~s}^{-1}$. This ionizing flux corresponds to that of a B3 ZAMS star with a luminosity

\footnotetext{
${ }^{8}$ The National Radio Astronomy Observatory is operated by Associated Universities Inc., under contract with the National Science Foundation.
}

of $1000 L_{\odot}$ (Thompson 1984). However, the IRAS luminosity of the associated source is only $\sim 10 L_{\odot}$. The phenomenon of low-luminosity young stars producing detectable thermal radio continuum emission is now known to be relatively common and has been recently discussed by Curiel et al. (1988). However, the detailed mechanism that produces the ionization is not yet established. Torrelles et al. (1985b) have argued that in the presence of stellar winds, shock-induced ionization could occur at levels comparable with what is observed.

The presence of radio continuum emission is suggestive of recent star-forming activity, and to futher investigate the core of $\mathrm{L} 1448$, we searched for $\mathrm{H}_{2} \mathrm{O}$ maser emission $(22.2 \mathrm{GHz})$ at the Haystack Observatory. We detected a powerful maser (Fig. 2) with position $\alpha(1950)=03^{\mathrm{h}} 22^{\mathrm{m}} 30^{\mathrm{s}} 8 \pm 1^{\mathrm{s}} 2$, $\delta(1950)=30^{\circ} 34^{\prime} 59^{\prime \prime} \pm 15^{\prime \prime}$. This position is coincident, within the uncertainties, with the location of the radio continuum source. The single feature observed can be fitted with a Gaussian profile having peak line flux, $S_{v}=729 \pm 2 \mathrm{Jy}$, half-power full width, $\Delta V=0.562 \pm 0.001 \mathrm{~km} \mathrm{~s}^{-1}$, and radial velocity with respect to the local standard of rest, $V_{\mathrm{LSR}}=3.337 \pm 0.001$ $\mathrm{km} \mathrm{s}^{-1}$. Assuming that the maser emits isotropically and that it is at a distance of $350 \mathrm{pc}$, we obtain an $\mathrm{H}_{2} \mathrm{O}$ luminosity of $L_{\mathrm{H}_{2} \mathrm{O}} \simeq 1.2 \times 10^{-6} L_{\odot}$. Since $L_{\mathrm{FIR}} \simeq 10 L_{\odot}($ Bachiller and Cernicharo 1986), we have $L_{\mathrm{H}_{2} \mathrm{O}} / L_{\mathrm{FIR}} \simeq 1.2 \times 10^{-7}$ for $\mathrm{L} 1448$. From the results of Wouterloot and Walmsley (1986), we esti-

TABLE 2

Line Parameters ${ }^{a}$ at Selected Positions of the Sources Detected in Ammonia

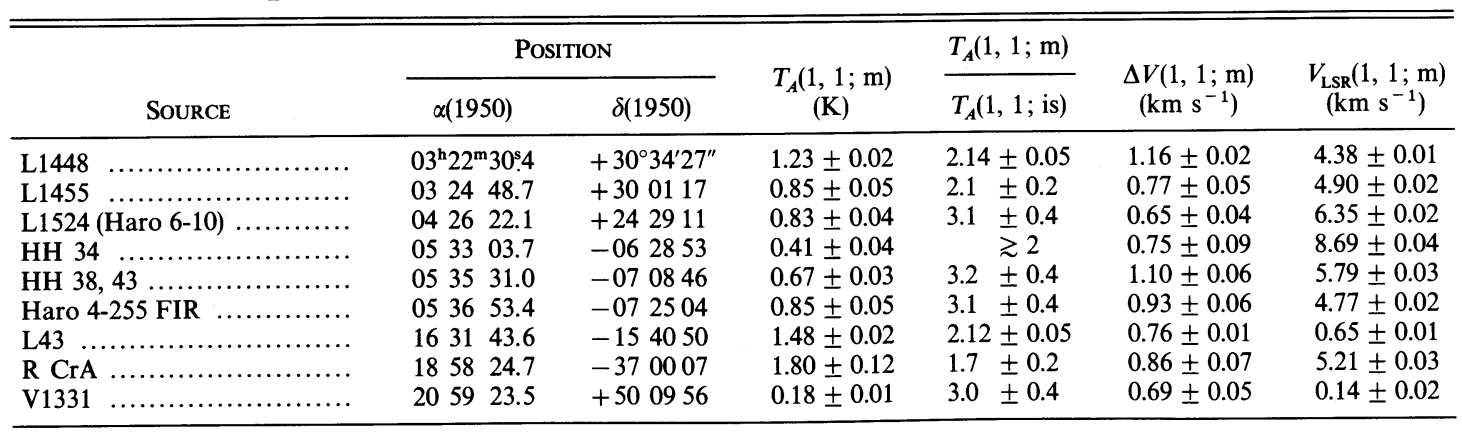

a Obtained from Gaussian fits to the observed spectra; $\mathrm{m}=$ main component $;$ is = inner satellite. 


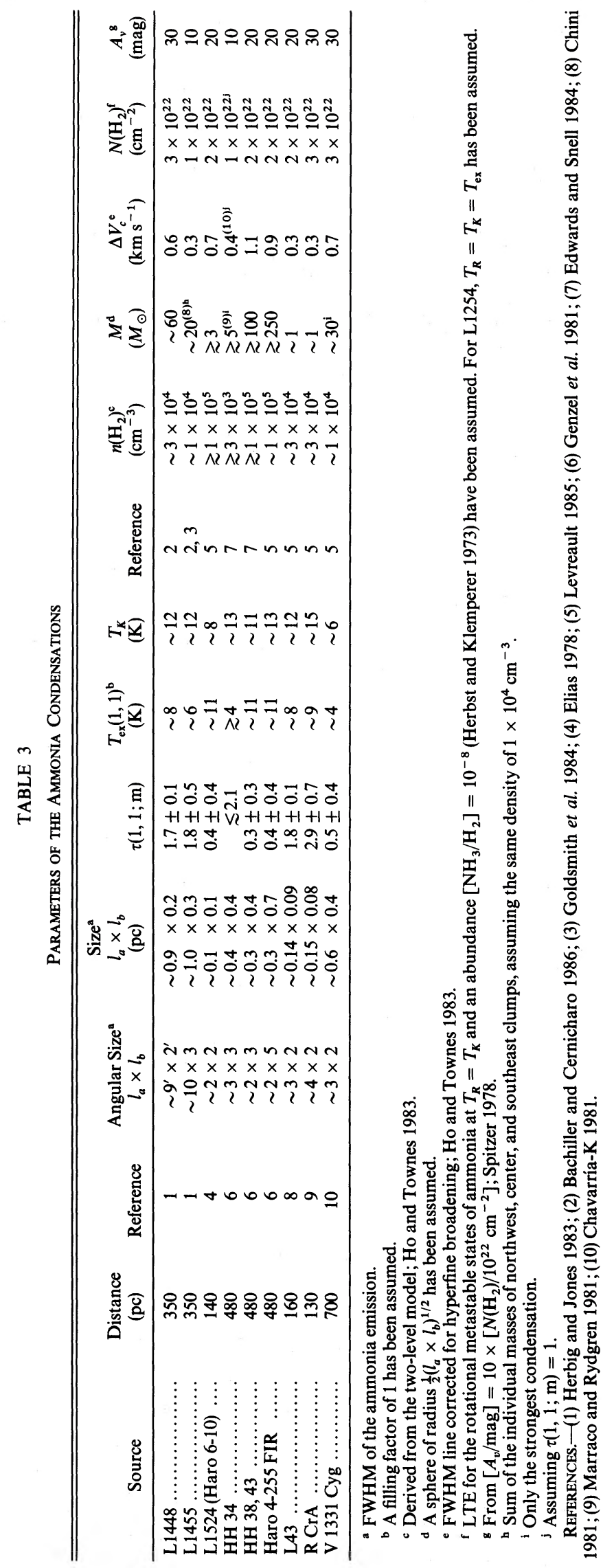

210 


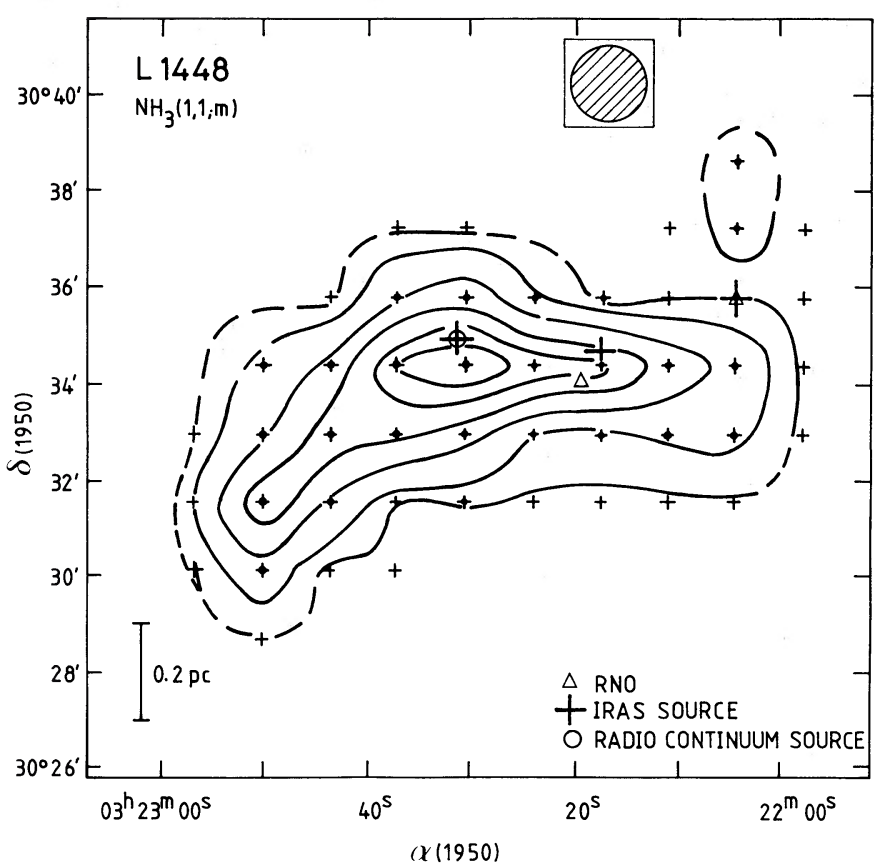

Fig. 1.-Contour map of the peak antenna temperature $T_{A}$ of the $(1,1 ; \mathrm{m})$ ammonia transition line for $\mathrm{L} 1448$. The observed positions are indicated with a small cross. Positions where ammonia has been detected are marked with a dot. The HPBW of the telescope is shown as a hatched circle. Contour levels are $0.1,0.2,0.4,0.6,0.8,1.0 \mathrm{~K}$.

mate that for galactic masers, typically $L_{\mathrm{H}_{2} \mathrm{O}} / L_{\mathrm{FIR}} \simeq 10^{-9 \pm 1}$. Then the $\mathrm{H}_{2} \mathrm{O}$ maser in $\mathrm{L} 1448$ is overluminous with respect to the region's FIR luminosity.

The elongated ammonia condensation shows a velocity shift of $\sim 0.8 \mathrm{~km} \mathrm{~s}^{-1}$, with the radial velocity decreasing from east to west. Bachiller and Cernicharo (1986) also found a velocity shift of $\sim 0.5 \mathrm{~km} \mathrm{~s}^{-1}$ between the east and west parts of the elongated condensation. It is not firmly established yet if this velocity shift is due to rotation or to the overlap of two clumps at different radial velocities.
The double-peaked profiles observed by Bachiller and Cernicharo (1986) appear only at the position of IRS 3 where the compact $\mathrm{H}$ II region and $\mathrm{H}_{2} \mathrm{O}$ maser are located. This result suggests that the double-peaked profile may be due to the pressure of a stellar wind, from the exciting source of the region, against the high-density molecular gas. A similar interpretation has been invoked for the HH 1-2 region (Torrelles et al. 1985a; Marcaide et al. 1988). In this way, we expect to find a gas outflow associated with the radio continuum source. This outflow could also be responsible for the shock-induced ionization mechanism for the $\mathbf{H}$ II region discussed before. Unfortunately, the CO observations of Levreault (1985) did not cover this region. We think that new $\mathrm{CO}$ observations centered at the $\mathrm{H}$ II region position could reveal the expected outflow.

We also did N-S cuts at $\alpha(1950)=03^{\mathrm{h}} 22^{\mathrm{m}} 31^{\mathrm{s}}$ in the $J=1 \rightarrow 0$ transition of $\mathrm{CS}(49 \mathrm{GHz})$ at the Haystack Observatory $\left(\mathrm{HPBW}=40^{\prime \prime}\right)$ and the $1_{10} \rightarrow 1_{01}$ transition of $\mathrm{C}_{3} \mathrm{H}_{2}(18$ GHz; Thaddeus, Vrtilek, and Gottlieb 1985) at the 140 foot $(42.7 \mathrm{~m})$ of the NRAO (HPBW $=1.6)$. The integrated line intensities from these cuts and that of $\mathrm{NH}_{3}$ are shown in Figure 3. The CS emission appears more extended than the $\mathrm{NH}_{3}$ emission. A similar discrepancy, with the CS emission being more extended than the ammonia (the $\mathrm{CS}$ and $\mathrm{NH}_{3}$ require similar critical densities of $\sim 10^{4} \mathrm{~cm}^{-3}$ for excitation) has also been found in other sources (e.g., HH 26-IR, Estalella et al. 1988; Cepheus A, Torrelles et al. 1987b; L43, Mathieu et al. 1988). This discrepancy is poorly understood. Fuller and Myers (1987) give a possible explanation in terms of scattering of the CS emission from the high-density core by CS molecules in a halo of relatively lower density. Morris et al. (1974) discuss a similar model in an attempt to account for the large sizes observed in $\mathrm{HCN}$ in some molecular clouds.

\section{b) $L 1455$}

This complex source has been mapped previously in ammonia by Schwartz, Frerking, and Smith (1985), Torrelles et al. (1986), and Bachiller and Cernicharo (1986). There is good agreement among all maps. Three IRAS sources, apparently associated with this molecular cloud, appear in the field. One

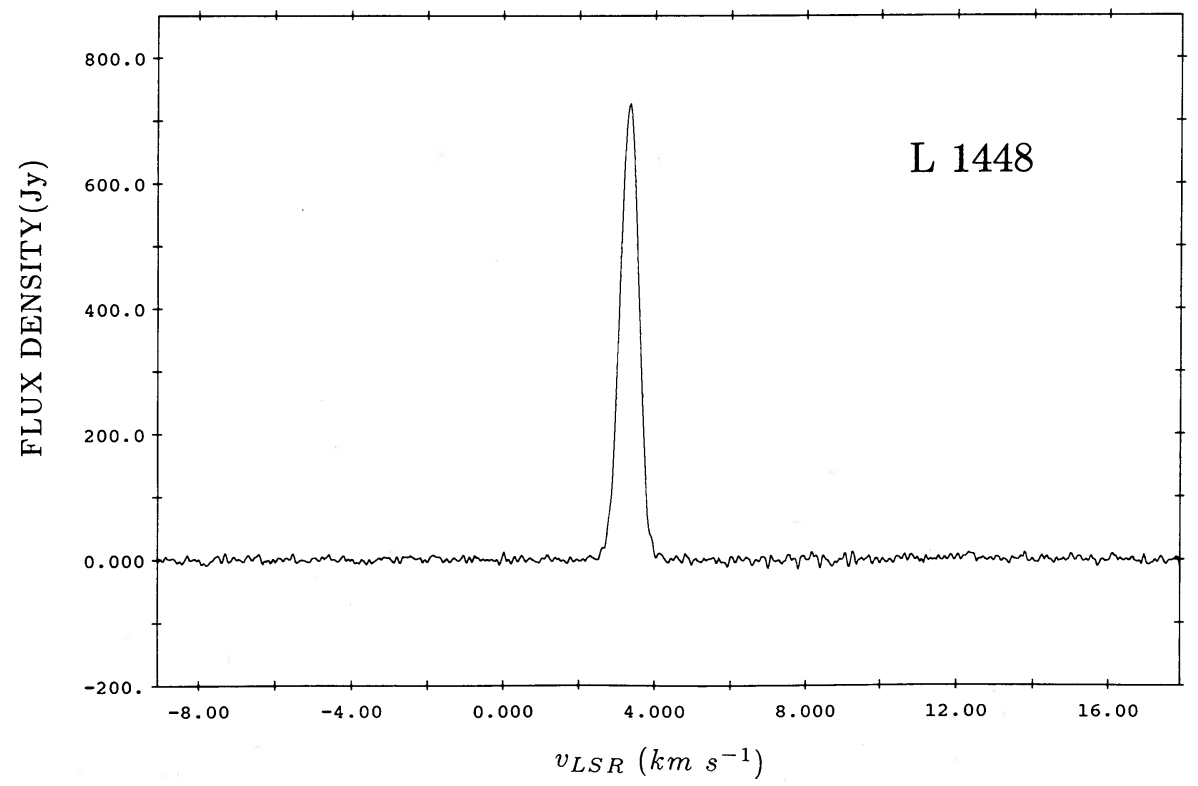

FIG. 2.-Spectrum of the water maser associated with the core of L1448 for the epoch 1988 July 31 . Velocity resolution is $0.066 \mathrm{~km} \mathrm{~s}^{-1}$. 


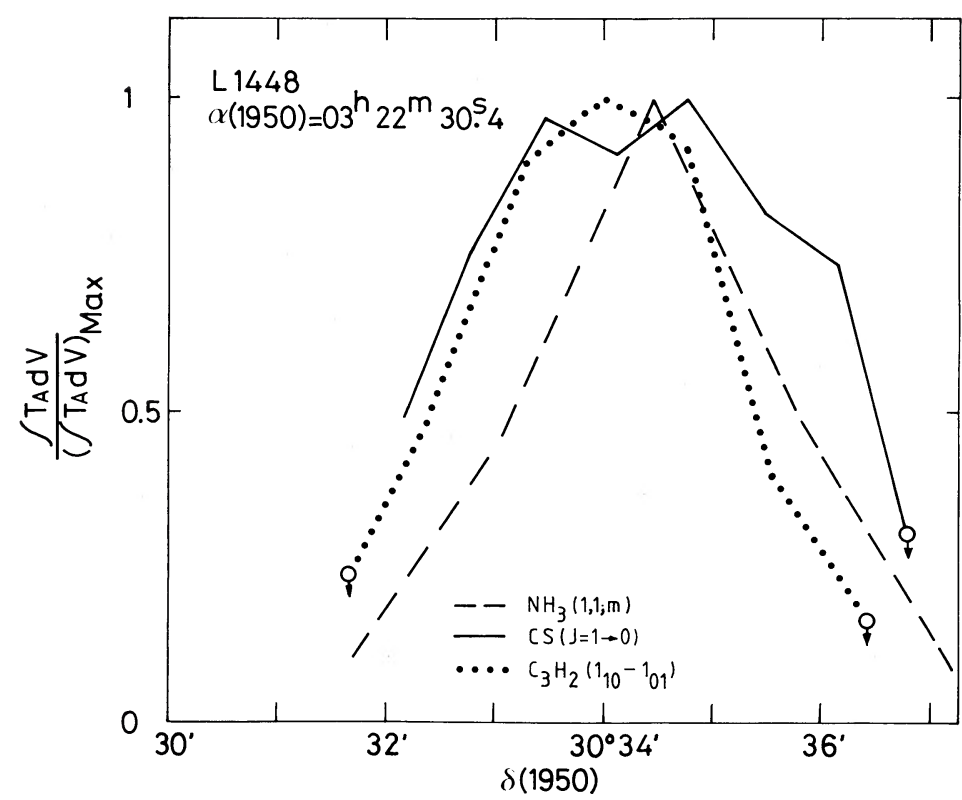

Fig. 3.-Normalized integrated line intensity of $\mathrm{NH}_{3}, \mathrm{CS}$, and $\mathrm{C}_{3} \mathrm{H}_{2}$ along the $\mathrm{N}-\mathrm{S}$ direction through the ammonia emission peak in $\mathrm{L} 1448$

of them (IRS 2) coincides with RNO 15. We mapped this region in order to better define the protuberances that appear in the SE-NW direction of the ammonia map of Torrelles et al. (1986). Our map is shown in Figure 4. Three clumps can be recognized in this figure. The strongest one peaks near RNO 15-IRS 2. The two other clumps are located where the protuberances detected by Torrelles et al. (1986) suggested a continuation of the ammonia emission. The southeast clump peaks near the IRS 3 object.
The CO outflow detected by Goldsmith et al. (1984) and Levreault $(1985,1988)$ shows multiple components of blueshifted and redshifted gas toward the central and NW condensations. However, these $\mathrm{CO}$ observations did not reach the area of the SE condensation, where high-velocity molecular gas associated with the high-density gas structure could also be present.

The clumpy ammonia structure suggests (as noted by Bachiller and Cernicharo 1986 for this case and by Torrelles et

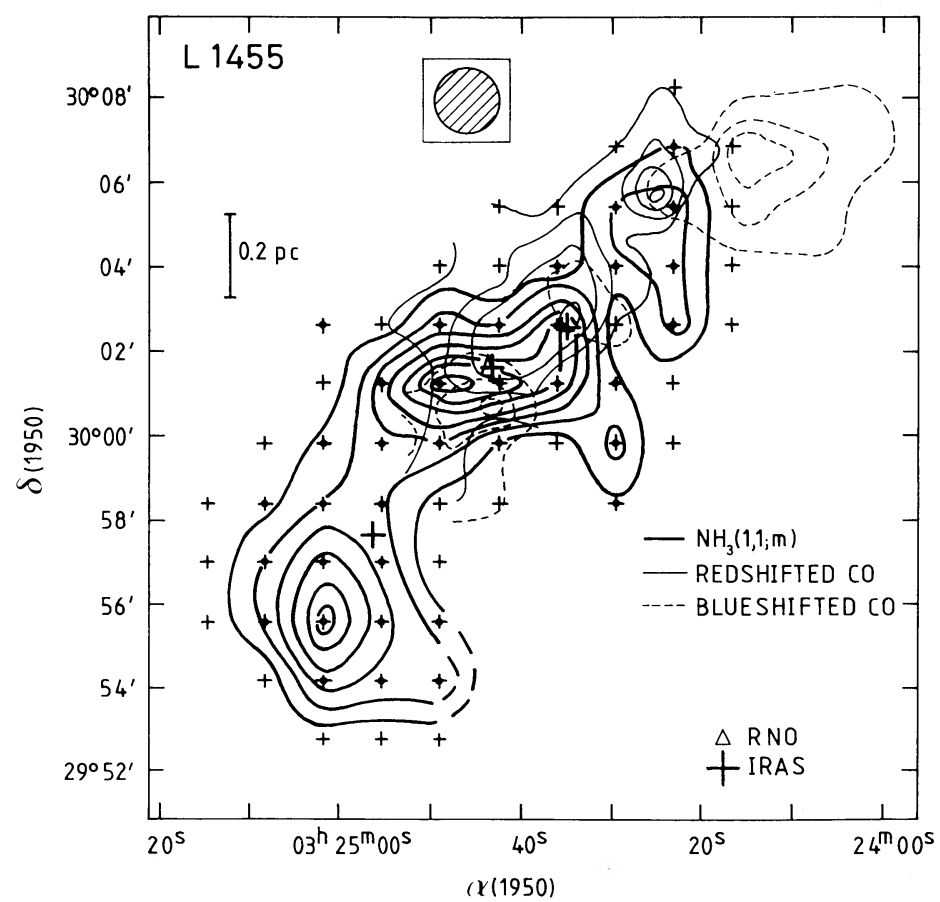

Fig. 4. - Same as Fig. 1 for L1455. The lowest contour level is $0.2 \mathrm{~K}$, and the increment is $0.1 \mathrm{~K}$. The CO outflow observed by Levreault (1985, 1988) is also shown superposed on the map. 
al. $1987 a$ for the HL/XZ Tau case) that the observed multiple $\mathrm{CO}$ outflows could be the result of a single outflow interacting in a complex manner with a clumpy medium.

\section{c) L1524 (Haro 6-10)}

This region contains the $\mathrm{H} \alpha$ emission star Haro 6-10 (Haro, Iriarte, and Chavira 1953). An extensive description of this object is given by Strom et al. (1986). These authors obtained $V, R, I$, and $\mathrm{H} \alpha \mathrm{CCD}$ images. Their images show that the star, which coincides with an IRAS source, is surrounded by a reflection nebulosity. They also found two small nebulosities in the $\mathrm{H} \alpha$ image. These two objects are located to the SW of Haro 6-10 and are roughly aligned with this star, suggesting an optical outflow with Haro 6-10 as the exciting source (Strom et al. 1986). Edwards and Snell (1984) and Levreault (1985) found slight indications of $\mathrm{CO}$ molecular outflow.

We mapped this region in ammonia in order to study the high-density gas around the possible exciting source Haro 6-10. In Figure 5, we show our map. The ammonia condensation peaks $\sim 2^{\prime} .5$ to the north of Haro 6-10, suggesting a possible new location for the exciting source. Our ammonia map is confirmed by the data of Benson and Myers (1989). However, recent $\mathrm{CS}(J=1 \rightarrow 0)$ observations carried out by Estalella et al. (1988) show a peak of emission toward Haro 6-10. Proper motion studies and spectroscopy of the two small nebulosities, as well as near-infrared and radio observations toward the ammonia clump to search for any embedded source, could give us an important clue for the confirmation of the optical outflow and the identification of the exciting source. It is important to emphasize that the lack of an $I R A S$ point source at the ammonia peak does not necessarily rule out the presence of the exciting source. This has been shown to be the case in HH 1-2 (Pravdo et al. 1985; Torrelles et al. 1985a) and HH 34 (Reipurth et al. 1986 and see below), where the exciting source is at an ammonia peak with no associated $I R A S$ point source.

\section{d) $\mathrm{RNO} 43$}

This region has been recently discussed by Cabrit, Goldsmith, and Snell (1988). These authors propose that its complex high-velocity pattern can be explained if the outflow is highly collimated and oriented nearly in the plane of the sky. We centered our nine-point grid on the RNO 43 object. We did not detect ammonia emission at a level of $T_{A} \geq 0.21 \mathrm{~K}$. Our grid did not reach the position of the infrared source located SW of RNO 43 and proposed by Jones et al. (1984) as the exciting source. We think that new observations covering the region around that infrared source could be relevant.

\section{e) $\mathrm{HH} 34$}

This region has been studied recently by Reipurth et al. (1986) and Strom et al. (1986). Edwards and Snell (1984) and Reipurth et al. (1986) could not detect evidence for a molecular outflow. On the other hand, Reipurth et al. (1986) discovered a highly collimated optical jet pointing toward $\mathrm{HH} 34$ and emanating from a faint star. HH 34 shows six knots embedded in a bow-shaped envelope. Recently, Bührke, Mundt, and Ray (1988) discovered a new Herbig-Haro object to the NW of the faint star, constituting a morphologically bipolar, optical outflow. Raga and Mateo (1988), through CCD images, estimate that an interstellar toroid with $\sim 10 M_{\odot}$ is needed to collimate the $\mathrm{HH} 34$ jet.

In Figure 6, we show our ammonia map. The peak of the strongest ammonia condensation coincides very closely with the faint star proposed as the exciting source by Reipurth et al. (1986). In Figure 7, we show the schematic representation of HH 34 given by Reipurth et al. (1986) superposed on the strongest ammonia condensation. The mass associated with

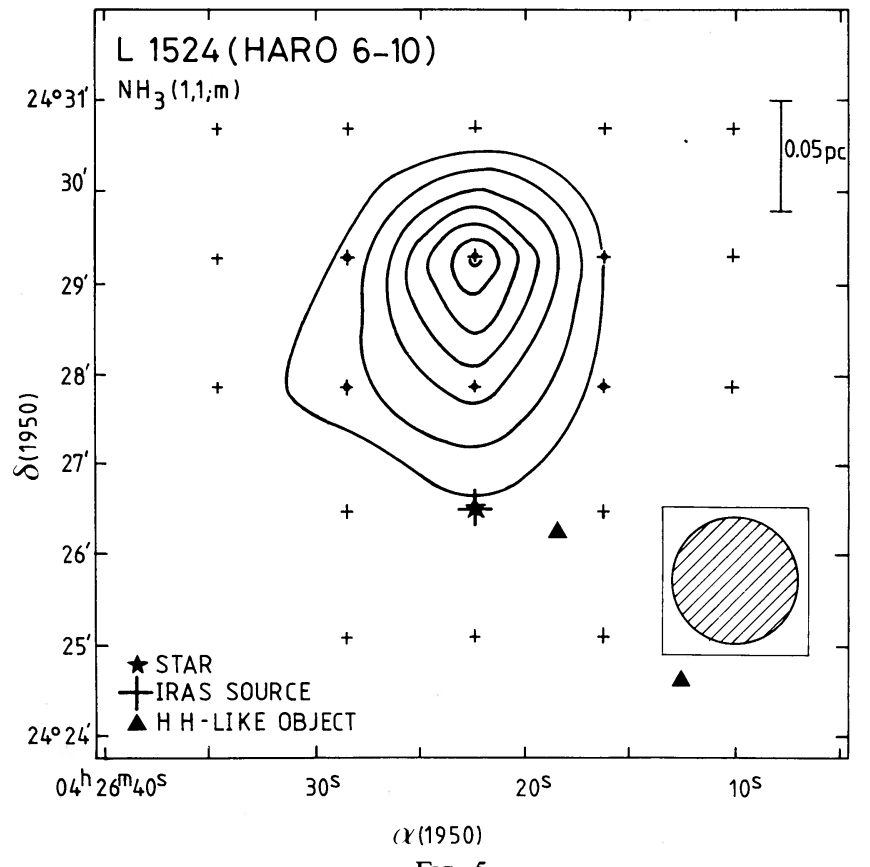

FIG. 5

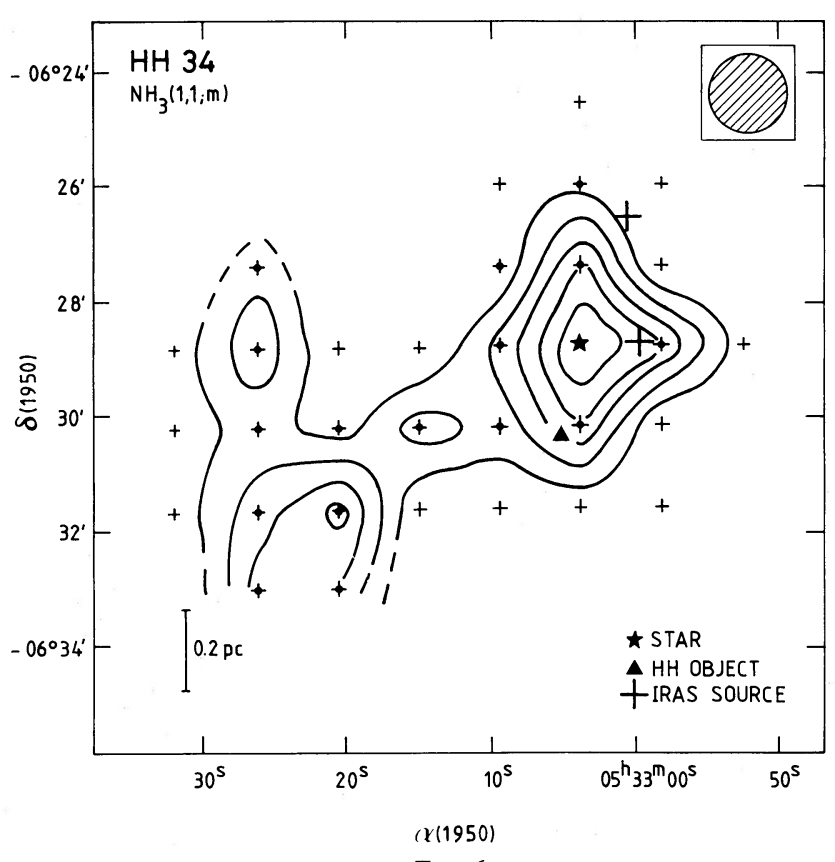

FIG. 6

Fig. 5.-Same as Fig. 1 for L1524 (Haro 6-10). The lowest contour level is $0.2 \mathrm{~K}$, and the increment is $0.1 \mathrm{~K}$.

Fig. 6.-Same as Fig. 1 for HH 34. The lowest contour level is $0.15 \mathrm{~K}$, and the increment is $0.05 \mathrm{~K}$. 


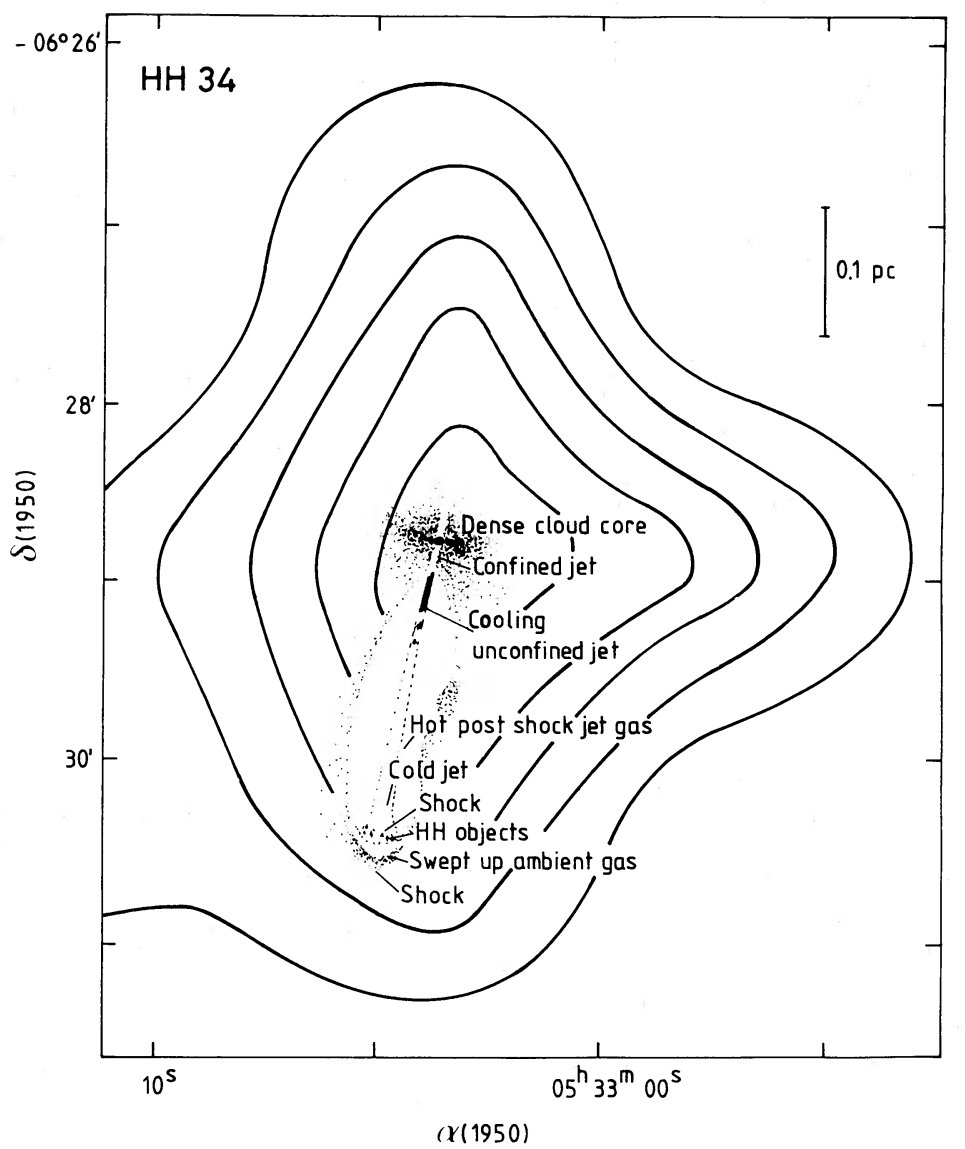

FIG. 7.-Schematic representation of HH 34 given by Reipurth et al. (1986) superposed on the strongest ammonia condensation (Fig. 6). The peak of the ammonia structure coincides with the proposed exciting source.

this condensation is $\gtrsim 5 M_{\odot}$, of the order of the mass of the toroid proposed by Raga and Mateo (1988). Unfortunately, our angular resolution is insufficient to spatially resolve the structure of the ammonia condensation and determine if it may be playing a role in the collimation processes. The ${ }^{13} \mathrm{CO}$ map of Reipurth et al. (1986) also peaks at the position of the proposed exciting star, and its morphology suggests that the gas may be distributed perpendicular to the direction of the star-HH objects axis.

\section{f) $\mathrm{HH} \mathrm{38,43}$}

HH 38 and HH 43 (Haro 1953; Herbig 1974) constitute a double $\mathrm{HH}$ object system that could be tracing an optical outflow (Cohen and Schwartz 1983). Edwards and Snell (1984) could not find convincing signs of well-ordered supersonic molecular motions in this region. An IRAS source, previously detected in the near-infrared by Cohen and Schwartz (1983), is located $\sim 1^{\prime} \mathrm{NW}$ from $\mathrm{HH} 43$. This infrared source was proposed by Cohen and Schwartz (1983) to be the exciting source of the $\mathrm{HH}$ objects 38 and 43 based on the alignment of the three objects along the same axis. Recently, Reipurth and Graham (1988) detected a new HH object, HH 64, in the region.

Our ammonia map is shown in Figure 8. The IRAS source and the $\mathrm{HH}$ objects 38 and 43 are located approximately at the SE border of the ammonia structure. The HH object 64 is located at the NW border of the ammonia structure. We think that the noncoincidence of the IRAS position with the high-

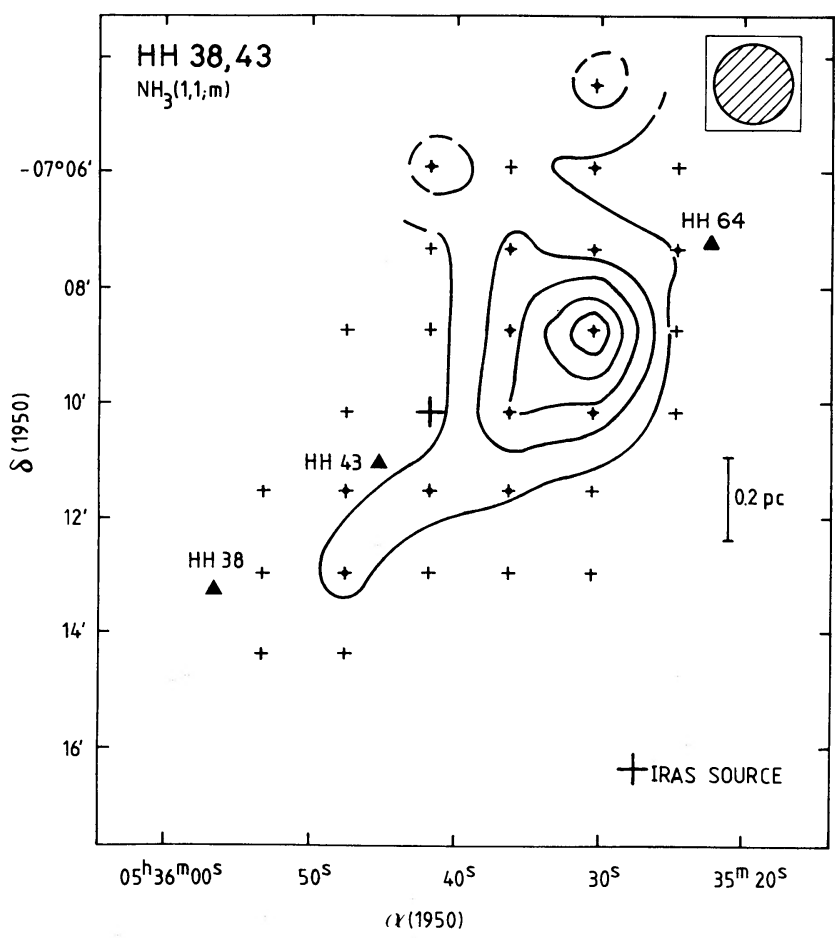

Fig. 8. - Same as Fig. 1 for $\mathrm{HH} \mathrm{38,} \mathrm{43.} \mathrm{The} \mathrm{lowest} \mathrm{contour} \mathrm{level} \mathrm{is} 0.15 \mathrm{~K}$, and the increment is $0.10 \mathrm{~K}$. 
density gas casts some doubt on the identification of the IRAS source as the exciting object of the possible optical outflow. Alternatively, the position of the ammonia clump, which is also located along the axis of the three $\mathrm{HH}$ objects, suggests that there can be an embedded source responsible for the excitation of the HH objects.

Proper motion studies of $\mathrm{HH} \mathrm{38,} \mathrm{HH} \mathrm{43,} \mathrm{and} \mathrm{HH} 64$ as well as near-infrared observations towards the ammonia clump, will help in clarifying the kinematics and the exciting source of these objects.

\section{g) Haro 4-255 FIR}

This far-infrared source (Levreault 1985, 1988) is located about $1^{\prime}$ to the NW of the $\mathrm{H} \alpha$ emission star Haro 4-255. A CO outflow approximately centered on Haro 4-255 FIR, suggesting a bipolar morphology, has been detected (Levreault 1985, 1988). Our ammonia map is dominated by two peaks of emission (Fig. 9). The position of the southern ammonia peak coincides with Haro 4-255 FIR, supporting the identification of the FIR source as the exciting source of the outflow. To our knowledge, there are no $\mathrm{CO}$ observations in the region around the northern ammonia condensation.

\section{h) NGC $2264(\mathrm{HH} \mathrm{14-4,6)}$}

We observed toward the position of the suspected exciting source (IRS 3) proposed by Cohen and Schwartz (1983). We did not detect ammonia at a level of $T_{A} \geq 0.36 \mathrm{~K}$. This result makes doubtful the identification of IRS 3 as the exciting source.

Further studies carried out by Cohen, Harvey, and Schwartz (1985) led them to propose an infrared source located $\sim 8^{\prime}$ south of the $\mathrm{HH}$ objects as a more plausible exciting source. New ammonia observations toward this particular position could help to confirm this new identification.

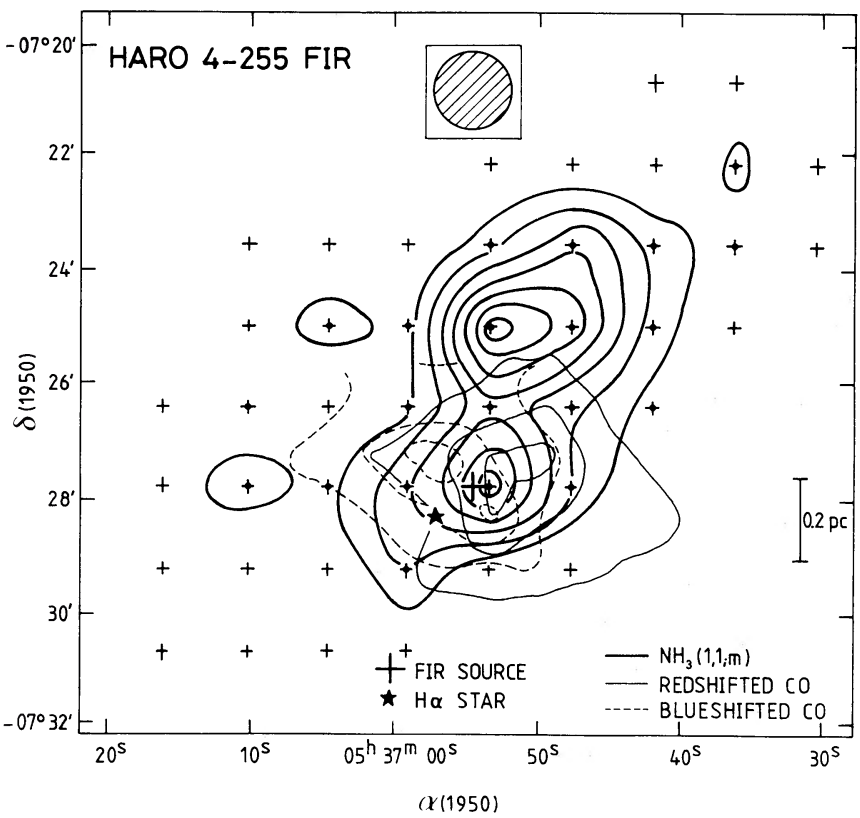

FIG. 9.-Same as Fig. 1 for Haro 4-255 FIR. The lowest contour level is 0.2 $\mathrm{K}$, and the increment is $0.1 \mathrm{~K}$. The CO outflow observed by Levreault (1985, 1988 ) is also shown superposed on the map.

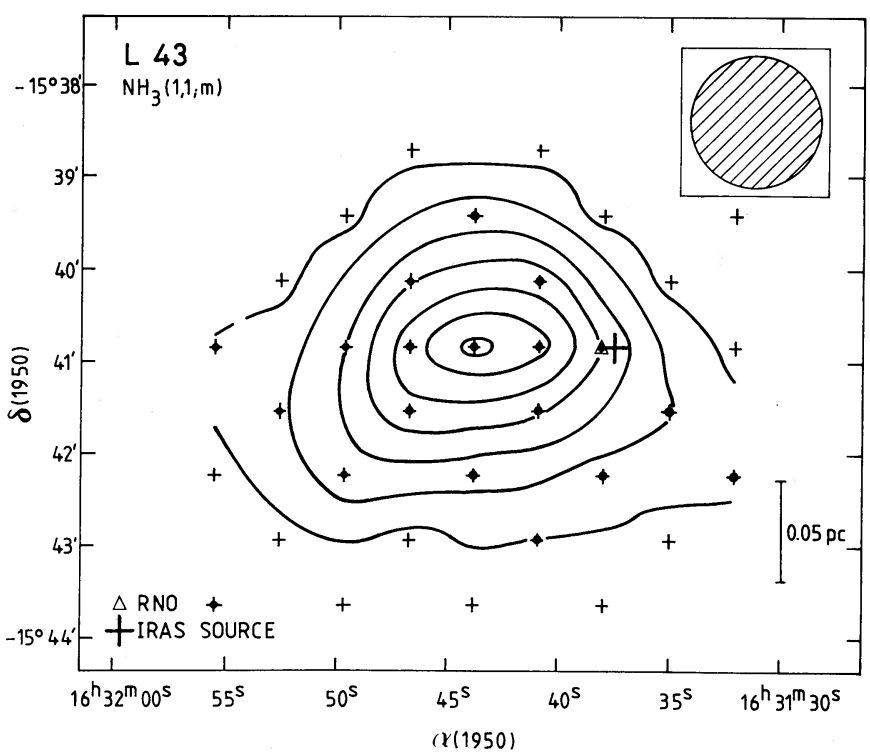

FIG. 10.-Same as Fig. 1 for L43. The lowest contour level is $0.2 \mathrm{~K}$, and the increment is $0.2 \mathrm{~K}$.

\section{i) $L 43$}

This region contains the RNO 90 and 91 objects. Levreault (1985) found a very weak monopolar outflow $\left(\Delta V=5 \mathrm{~km} \mathrm{~s}^{-1}\right.$ at $0.2 \mathrm{~K}$ level) with only blueshifted wings present, and apparently associated with RNO 91 . This outflow is extended in the south direction. Myers et al. (1988) suggest that the outflow is bipolar.

In Figure 10, we show our ammonia map. The peak of the ammonia condensation is displaced $\sim 1^{\prime} .5$ to the east of RNO 91. To the SW, there is still emission at a distance of $2^{\prime}$ from RNO 91, where our map terminates. This suggests a more extended emission, coinciding with the ammonia condensation recently reported by Mathieu et al. (1988). Their data also show the strongest ammonia condensation to the east of RNO 91, in excellent agreement with ours. These authors show CCD images revealing an optical bay around RNO 91, coinciding with the blueshifted $\mathrm{CO}$ outflow. They also observe an increase of the line width of the ammonia emission within the bay from east to west towards RNO 91. All these results have been interpreted as the action of the stellar wind associated with RNO 91 that has cleared out the high-density gas of its near environment (Mathieu et al. 1988).

\section{j) $\mathrm{R} \mathrm{Cr} A$}

This star is at the head of the cometary nebula NGC 6729. It is associated with an important concentration of molecular gas (Loren 1979). Two different outflows, one molecular and the other optical, seem to be present in the region. The molecular outflow, traced by the $\mathrm{CO}$, and apparently associated with $\mathrm{R}$ CrA, shows a very complex morphology (Levreault 1985). While the outflow core is aligned almost east-west, its outer parts are aligned with a position angle of about $30^{\circ}$. Proper motion studies on the HH 99 and HH 101 objects (Schwartz, Jones, and Sirk 1984; Hartigan and Graham 1987) show an optical bipolar outflow, with HH 99 and HH 101 moving in opposite directions with respect to the proposed exciting source HH 100 IR (Strom, Grasdalen, and Strom 1974) with a position angle of $30^{\circ}$. This bipolar outflow resembles that of 
HH 1-2 (Herbig and Jones 1981; Pravdo et al. 1985; Rodríguez et al. 1989).

We show in Figure 11 our ammonia map. The core of the $\mathbf{R}$ CrA outflow does not appear to correlate with the ammonia structure (see Fig. 11 [bottom]). However HH 100 IR is located very close to the southern ammonia peak. In Figure 12, we show the ammonia map superposed on the optical image obtained by Hartigan and Graham (1987). We can appreciate that the high-density structure coincides with the obscured region around HH 100 IR. From our ammonia data, we estimate for this region a visual extinction of $A_{v} \sim 30$ mag (Table 3). This implies that the reflection nebulae NGC 6729 and NGC 6726/7 (coinciding with the northern ammonia peak) are probably located in front of the ammonia core. These results suggest that we have detected the high-density gas associated with the HH 99-HH 101 bipolar outflow, giving support to HH 100 IR as the exciting source. In this sense, the core of the molecular outflow may be related to molecular gas of relatively lower density located at the surface of the cloud mapped by Loren (1979). A similar phenomenon is found in the $R$ Mon cometary nebula (Cantó et al. 1981). The similar position angles of the outer parts of the molecular outflow and the optical bipolar outflow lead us to propose that the stellar wind associated with the suspected exciting source HH 100 IR also produces a molecular outflow in the direction of $\mathrm{HH} 99$ and HH 101. The overlap of the east-west molecular outflow,

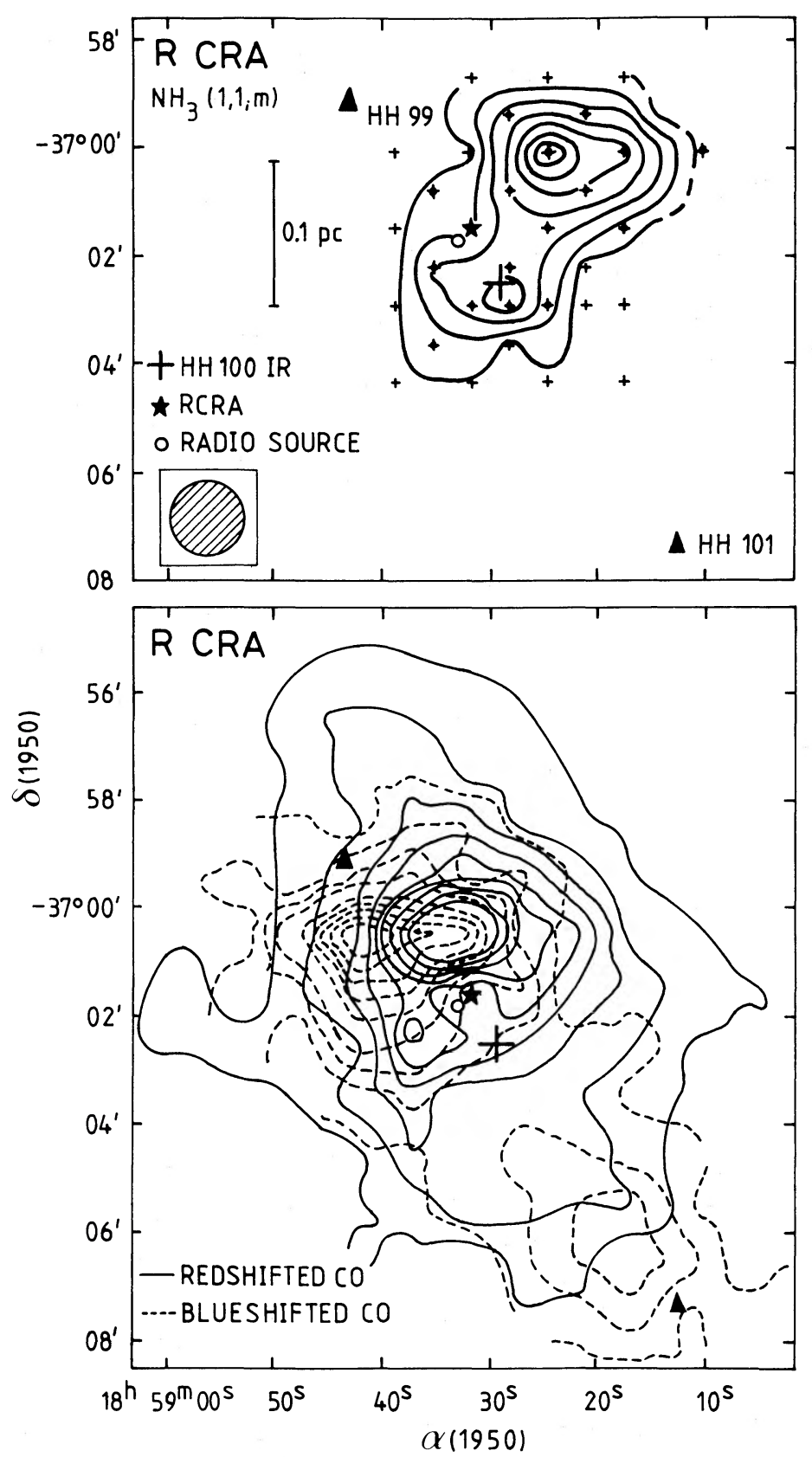

Fig. 11. - (top) Same as Fig. 1 for R CRA. The lowest contour level is $0.3 \mathrm{~K}$, and the increment is $0.2 \mathrm{~K}$. (bottom) CO outflow observed by Levreault (1985, 1988). 


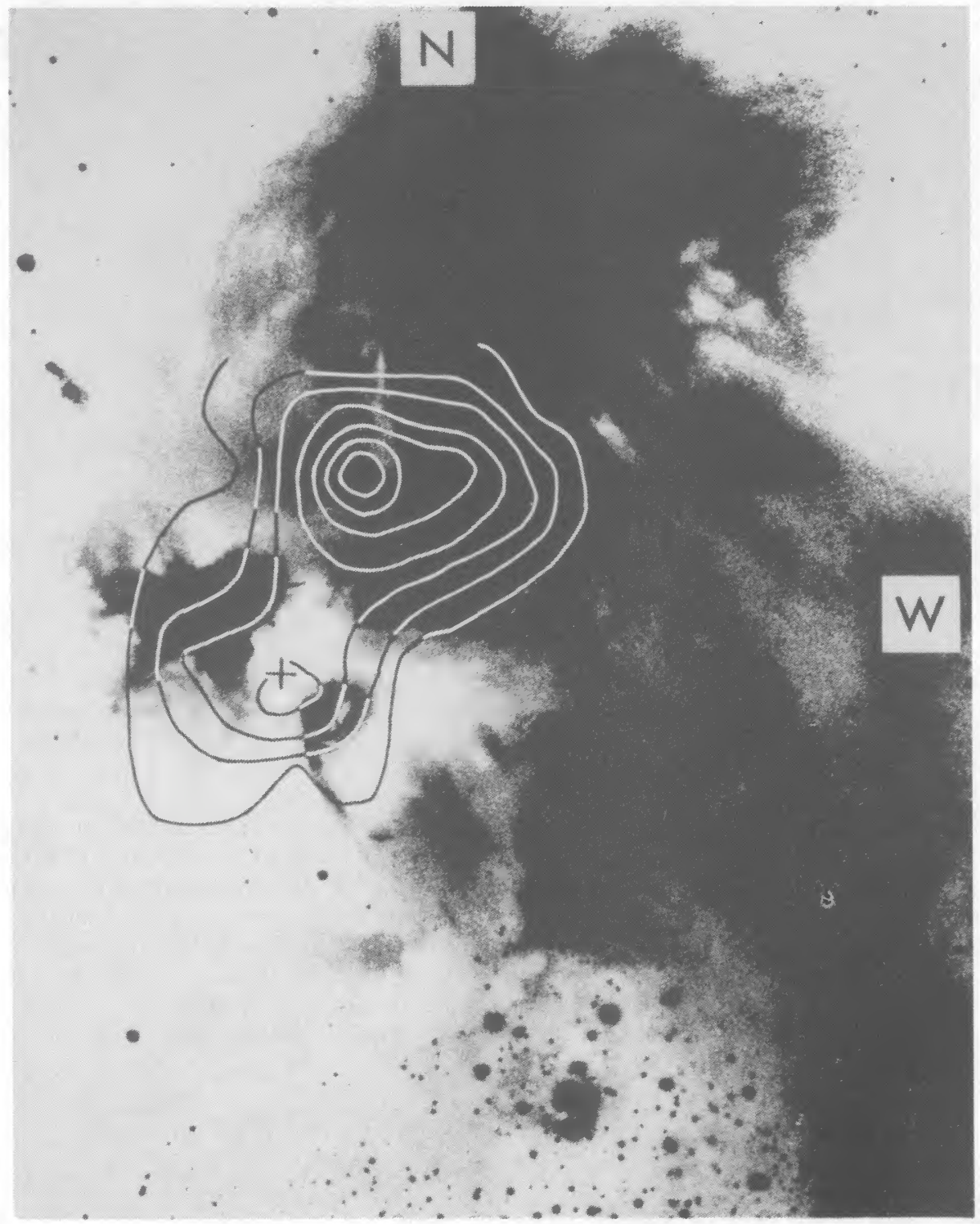

Fig. 12.-Ammonia map (Fig. 11 [top]) superposed on a negative print of the R CrA region at a wavelength of $655 \mathrm{~nm}$ taken by Hartigan and Graham (1987)

associated with NGC 6729, with the NE-SW molecular outflow, associated with $\mathrm{HH} 100 \mathrm{IR}$, could explain the complex morphology observed by Levreault (1985).

Finally, it is worth noting that there is a compact radio source in the region (Cruz-González, McBreen, and Fazio 1984) that coincides positionally (within $2^{\prime \prime}$ ) with the R1 nearinfrared source studied by Wilking, Taylor, and Storey (1986). This IR source has a bolometric luminosity of a few $L_{\odot}$ and cannot provide the ionizing photons required by the radio source, assuming that it is an $\mathrm{H}$ II region. It appears that $\mathrm{R} 1$ could be another example of a low-luminosity young star that can produce significant amounts of ionization (Curiel et al. 1988). Recently, Brown (1987) detected several other radio sources in the $\mathrm{R} \mathrm{Cr}$ A region.

\section{k) $\mathrm{HH} 32 \mathrm{a}(\mathrm{AS} 353 \mathrm{~A})$}

Cohen and Schwartz (1983) proposed AS 353A as the exciting source of the $\mathrm{HH}$ object. Torrelles et al. (1983) failed to detect ammonia emission at the position of AS 353A. To see if any ammonia emission existed beyond AS 353A, we made new observations at the positions of a nine-point grid. We did not detect ammonia emission at a level of $T_{A} \geq 0.18 \mathrm{~K}$.

\section{l) V1331 Cyg}

Levreault (1985) reported a very weak monopolar (redshifted) outflow $\left(\Delta V=7 \mathrm{~km} \mathrm{~s}^{-1}\right.$ at $0.2 \mathrm{~K}$ level) associated with this star and stretching in the south direction. Our ammonia map (Fig. 13) shows two condensations. The strongest one peaks near V1331 Cyg, where there is also an IRAS point source.

\section{CONCLUSIONS}

We have observed with the $37 \mathrm{~m}$ telescope of the Haystack Observatory 12 sources with molecular or optical outflows in the $(J, K)=(1,1)$ ammonia transition. We have detected and mapped the ammonia emission in nine of these sources. Our main conclusions can be summarized as follows.

1. In five of the detected sources (HH 34, Haro 4-255 FIR, 


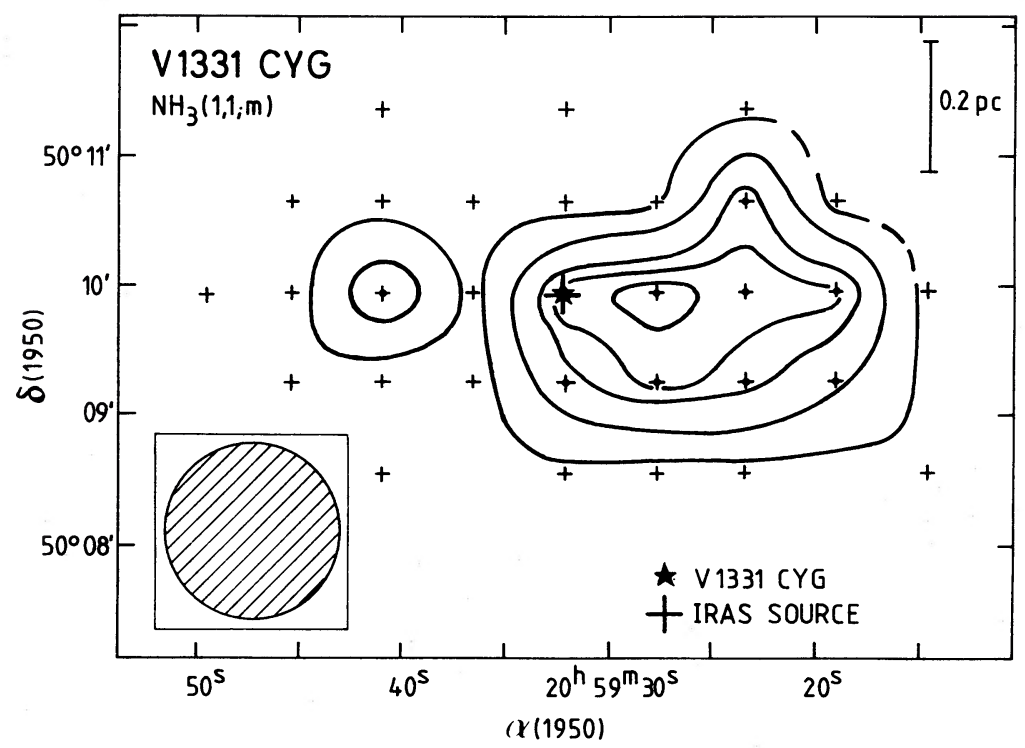

Fig. 13. - Same as Fig. 1 for V1331 Cyg. The contour levels are $0.07,0.11,0.15,0.17,0.19 \mathrm{~K}$.

L43, R CrA, and V1331 Cyg), the ammonia emission peaks near the objects previously proposed to be the exciting source. Our results support these identifications.

2. In two of the detected sources (L1524 and HH 38, 43), the ammonia emission peaks about $3^{\prime}$ off the previously proposed exciting source. The detected clumps are roughly aligned with the axis defined by the HH-like nebulosities or the HH objects. These results suggest a new location near the ammonia peak for the outflow exciting source.

3. In L1448, we also detected a radio continuum source and a strong water maser at the peak of the ammonia emission. There is also an IRAS source at this position. The observed double-peaked profiles of different molecular lines toward the radio continuum source and the possibility of shock-induced ionization lead us to suggest the presence of a high-velocity gas outflow associated with the detected central object.

We thank P. Hartigan for providing us with the optical image of the R CrA region and P. F. Goldsmith for his careful reading of the manuscript. We are most grateful to $\mathbf{J}$. M. Moran for his hospitality. G. A. acknowledges partial financial support from CIRIT de Catalunya, Spain. G. A., R. E., R. L., J. M. T., and L. V. acknowledge partial financial support from SEUI (Spain) grant PB0371. J. M. T. also acknowledges the Junta de Andalucía, Spain for partial financial support.
Adams, M. T., Strom, K. M., and Strom, S. E. 1979, Ap. J. (Letters), 230, L183.

Bachiller, R., and Cernicharo, J. 1986, Astr. Ap., 168, 262.

Benson, P., and Myers, P. C. 1989, Ap. J. Suppl., in press

Brown, A. 1987, Ap. J. (Letters), 322, L31.

Bührke, T., Mundt, R., and Ray, T. P. 1988, Astr. Ap., 200, 99

Cabrit, S., Goldsmith, P. F., and Snell, R. L. 1988, Ap. J., 334, 196.

Cantó, J. 1985, in Cosmical Gas Dynamics, ed. F. D. Kahn (Utrecht: VNU Science Press), p. 267.

Cantó, J., Rodríguez, L. F., Barral, J. F., and Carral, P. 1981, Ap. J., 244, 102.

Chavarria-K. C. 1981, Astr. Ap., 101, 105.

Chini, R. 1981, Astr. Ap., 99, 346.

Cohen, M. 1980, A.J., 85, 29 .

Cohen, M., Bieging, J. H., and Schwartz, P. R. 1982, Ap. J., 253, 707.

Cohen, M., Harvey, P. N., and Schwartz, R. D. 1985, Ap. J., 296, 633.

Cohen, M., and Schwartz, R. D. 1983, Ap. J., 265, 877.

Cruz-González, I., McBreen, B., and Fazio, G. G. 1984, Ap. J., $279,679$.

Curiel, S., Rodríguez, L. F., Cantó, J., Bohigas, J., Roth, M., and Torrelles,

J. M. 1988, Ap. Letters Comm., 27, in press.

Davidson, J. A., and Jaffee, D. T., 1984, Ap. J. (Letters), 277, L13.

Edwards, S., and Snell, R. L. 1982, Ap. J., 261, 151.

. 1984, Ap.J., 281, 237.

Elias, J. H. 1978, Ap. J., 224, 857

Estalella, R. et al. 1989 , in preparation.

Evans, N. J., Levreault, R. M., and Harvey, P. M. 1986, Ap. J., 301, 894.

Fuller, G. A., and Myers, P. C. 1987, in Physical Processes in Interstellar Clouds, ed. G. Morfill and M. Scholer (Dordrecht: Reidel), in press.

Genzel, R., Reid, M. J., Moran, J. M., and Downes, D. 1981, Ap. J., 244, 884.

Goldsmith, P. F., Snell, R. L., Hemeon-Heyer, M., and Langer, W. D. 1984, Ap. $J ., 286,599$.

González, G., and González, G. 1956, Bol. Obs. Tonantzintla y Tacubaya, 15, 16.

Haro, G. 1953, Ap. J., 117, 73.

. 1959, private communication to G. H. Herbig.

Haro, G., Iriarte, B., and Chavira, E. 1953, Bol. Obs. Tonantzintla y Tacubaya, 8,3 .

\section{REFERENCES}

Hartigan, P., and Graham, J. A. 1987, A.J., 93, 913.

Herbig, G. H.1960, Ap. J. Suppl., 6, 337.

. 1974, Lick Obs. Bull., No. 658.

Herbig, G. H., and Jones, B. F. 1981, A.J., 86, 1232.

. 1983, A.J., 88, 1040 .

Herbig, G. H., and Kuhi, L. V. 1963, Ap. J., 137, 398.

Herbst, E., and Klemperer, W. 1973, Ap. J., 185, 505.

Ho, P. T. P., and Townes, C. H. 1983, Ann. Rev. Astr. Ap., 21, 239.

Jones, B. F., Cohen, M., Sirk, M., and Janett, R. 1984, A.J., 89, 1404.

Lada, C. J. 1985, Ann. Rev. Astr. Ap., 23, 267.

Levreault, R. M. 1985, Ph.D. thesis, University of Texas at Austin.

. 1988, Ap. J. Suppl., 67, 283.

Little, L. T., Dent, W. R. F., Heaton, B., Davies, S. R., and White, G. J. 1985 M.N.R.A.S., 217, 227.

Loren, R. B. 1979, Ap. J., 227, 832

Marcaide, J. M., Torrelles, J. M., Güsten, R., Menten, K., Ho, P. T. P., Moran, J. M., and Rodríguez, L. F. 1988, Astr. Ap., 197, 235.

Marraco, H. G., and Rydgren, A. E. 1981, A.J., 86, 62.

Mathieu, R. D., Benson, P. J., Fuller, G. A., Myers, P. C., and Schild, R. E. 1988, Ap. J., 330, 385.

Morris, M., Palmer, P., Turner, B. E., and Zuckerman, B. 1974, Ap. J., 191, 349.

Mundt, R. 1986, Canadian J. Phys., 64, 407.

Myers, P. C., Heyer, M., Snell, R. L., and Goldsmith, P. F. 1988, Ap. J., 324, 907.

Pravdo, S. H., Rodríguez, L. F., Curiel, S., Cantó, J., Torrelles, J. M., Becker, R. H., and Sellgren, K. 1985, Ap. J. (Letters), 293, L35.

Raga, A. C., and Mateo, M. 1988, A.J., 95, 543.

Reipurth, B., Bally, J., Graham, J. A., Lane, A., and Zealey, W. J. 1986, Astr. Ap., 164, 51 .

Reipurth, B., and Graham, J. A. 1988, Astr. Ap., 202, 219.

Rodríguez, L. F., Carral, P., Ho, P. T. P., and Moran, J. M. 1982, Ap. J., 260, 635 .

Rodríguez, L. F., Ho, P. T. P., Torrelles, J. M., Curiel, S. and Canto, J. 1989, in preparation.

Schwartz, P. R., Frerking, M. A., and Smith, H. A. 1985, Ap. J., $295,89$. 
Schwartz, P. R, Waak, J. A and Smith, H. A. 1983, Ap. J. (Letters), 267, L109. Schwartz, R. D. 1983, Ann. Rev. Astr. Ap., 21, 209.

Schwartz, R. D., Jones, B. F., and Sirk, M. 1984, A.J., 89, 1735

Spitzer, L. 1978, Physical Processes in the Interstellar Medium (New York: John Wiley \& Sons).

Strom, K. M., Strom, S. E., Wolff, S. C., Morgan, J., and Wenz, M. 1986, Ap. J. Suppl., 62, 39.

Strom, S. E., Grasdalen, G. L., and Strom, K. M. 1974, Ap. J., 191, 111

Takano, T., Stutzki, J., Fukui, Y., and Winnewisser, G. 1987, in IAU Symposium 115, Star Forming Regions, ed. M. Peimbert and J. Jugaku (Dordrecht: Reidel), p. 361.

Thaddeus, P., Vrtilek, J. M., and Gottlieb, C. A. 1985, Ap. J. (Letters), 299, L63.

Thompson, R. I. $1984, A p$. J., 283, 165.

Torrelles, J. M., Anglada, G., Rodríguez, L. F., Cantó, J., and Barral, J. F. 1987a, Astr. Ap., $177,171$.
Torrelles, J. M., Cantó, J., Rodríguez, L. F., Ho, P. T. P., and Moran, J. M. 1985a, Ap. J. (Letters), 294, L117.

Torrelles, J. M., Ho, P. T. P., Moran, J. M., Rodríguez, L. F., and Cantó, J. 1986, Ap. J., 307, 787.

Torrelles, J. M., Ho, P. T. P., Rodríguez, L. F., and Cantó, J. 1985b, Ap. J., 288 595.

Torrelles, J. M., Ho, P. T. P., Rodríguez, L. F., Cantó, J., and Moran, J. M. 1987b, Ap. J., 321, 884.

Torrelles, J. M., Rodríguez, L. F., Cantó, J., Carral, P., Marcaide, J., Moran, J. M., and Ho, P. T. P. 1983, Ap. J., 274, 214.

Wilking, B. A., Taylor, K. N. R., and Storey, J. W. V. 1986, A.J., 92, 103

Wouterloot, J. G. A., and Walmsley, C. M. 1986, Astr. Ap., 168, 237.

Guillem Anglada, Robert Estalella, and Rosario López: Departament de Física de l'Atmosfera, Astronomia i Astrofísica, Universitat de Barcelona, Diagonal 645, E-08028 Barcelona, Spain

Jorge Cantó: Instituto de Astronomía, UNAM, Apdo. Postal 70-264, 04510, México, D. F., Mexico

Paul T. P. Ho and LuIS F. Rodríguez: Harvard-Smithsonian Center for Astrophysics, 60 Garden Street, Cambridge, MA 02138

José M. Torrelles and Lourdes Verdes-Montenegro: Instituto de Astrofísica de Andalucía, CSIC, Apdo. Correos 2144 , E-18080 Granada, Spain 